\title{
Editorial
}

\section{Facing up to the year 2000 computer bug}

If you take one message away from this article about the year 2000 computer problem it should be "panic now"; or better still, translate your panic into constructive lobbying and action to avoid the computer problems that will occur at the start of the millennium! Experts agree that every medical discipline will be affected, ${ }^{1}$ and pathology, with the emphasis on storage, analysis, retrieval, and manipulation of clinical data, will be one of the main casualties of the millennium bug. The problem is not simply one for the most IT literate in your laboratory, hospital or medical school to identify and plan (and fund) the solutions; it will be a major organisational issue that requires senior management input. ${ }^{2}$ Even with advanced planning and remedial action, there will inevitably be computer related problems on 1 January 2000 . It would be careless, and probably negligent, not to attempt to anticipate as many as problems as possible.

In this short article, I will give an overview of the history of the millennium problem, some of the likely consequences, and practical ideas of how to begin to identify where they might occur. Obviously, each department will have specific problems and therefore unique solutions; identifying some of these problems and finding the solutions might be very straightforward or technically very difficult. $^{3}$ Detailed advice is beyond the scope of this article, nevertheless, if reading this frightens you into encouraging your department, hospital or medical school to take the problem seriously, it will have achieved its aim.

\section{History of the millennium bug}

The central element of the year 2000 computer problem is what will happen when, at the stroke of midnight on the last day of 1999, the date on the computer tries to change to 2000. The difficulties stem from the earliest days of computing, when data was stored on punched Hollerith cards or in the small, and extremely expensive, electronic memory of the first computers. To save storage space in both these systems, programmers truncated the year to the last two digits: 1964 became 64. Even when computer memory became cheaper, many programmers stuck to their old ways and continued to write programs with just two digits representing the date. This continued into the early 1990s; programmers reasoning that the advance of computer technology was so fast that a problem nearly a decade away was irrelevant.

As with all computer problems, there are issues related to computer hardware and others that are a function of the software; some millennium problems arise because of a combination of the two and these are among the most unpredictable.

\section{Interpreting the date change}

The odometer problem describes what occurs when the hardware in your computer interprets dates after $1999 .{ }^{3}$ Unlike a car, where the odometer will invariably change from a string of $9 \mathrm{~s}$ to a string of $0 \mathrm{~s}$, computers are less predictable in the way they set their dates. What the date becomes depends upon the choice of internal silicon chips and the software running on them. Some computers will change from 1999 to 2000, but others might become 1900, 1980 or even 1984 . This is relatively easy to check - set the system clock on your computer to 31.12.1999 at 23:59:51, leave it to run for 10 seconds and see what the display shows. Before you relax, you should bear in mind that even if it becomes 01.01 .00 , there is no certainty that this is not 01.01 .1990 or 01.01.1980, so it is essential to create a file and look at the actual date on that file. Even then you are far from home and dry-certain programs use the internal hardware clock in some situations, while in others they calculate the date by internal reference in the program itself. To make matters worse, if your system is networked, certain programs will use the clock on the main fileserver to determine the date on some occasions, while on others it will be the clock on the client computers.

Finding how computer software interprets the date is far from trivial, but there are a variety of programs available to run this type of test, and many are available free on the internet. ${ }^{4}$ Even with these, the most difficult and costly software to analyse are the programs that have been written in-house, say by a passing summer student or an enthusiastic member of staff who has left or retired. In some of these, each line of computer code must be checked and understood; often any documentation is skimpy and the program structure obscure.

\section{Embedded systems and dates}

The problem does not end with computer systems and software. We take for granted many highly sophisticated laboratory machines such as autoanalysers, flow cytometers, and embedding machines; all of these have silicon chips in their control circuits (so called embedded systems). Many know the date and time, and some are designed to shut down automatically at certain dates if they are not serviced or have their software upgraded. The Institute of Electronic Engineers has just produced guidance on investigating these systems. ${ }^{5}$

Dates on system clocks and within the specific operating system hardware of a computer are, to a limited degree, predictable because, with a little thought, they can be found and tested. Much more alarming are the so called embedded dates, those dates hidden within program software code or buried within other information. For example, the UK driving licence number contains information on the owner's date of birth, but with the digits jumbled making it obscure to the casual viewer, but these data are essential to a computer wishing to calculate when the licence expires. It is not hard to imagine how difficult it will be to identify these scrambled numbers in the millions of lines of software code that operate behind the scenes to organise and manage our everyday lives.

Surviving the first day of the new millennium will not be the end of the problem. Many of the glitches will only begin to appear months or even years into the new century as the effect of erroneously calculated dates emerge. For example, what will happen in the year 2000 if your cervical screening recall system calculates reminder dates by reference to the 
year 1900 or 1984 ? Some computer back up systems will overwrite newer files with older ones because they fail to understand that the file with year date 99 is older than that with the year ending 00. All your medical students may be prevented from sitting their examinations because the computer believes they have entered 99 years too late!

Is your hospital or medical school prepared for 1 January 2000 ? Is there a senior management committee addressing the problem? Have the resource implications of an estimated $5 \%$ to $25 \%$ of your annual IT expenditure been identified? ${ }^{6}$ Has a group with the necessary (and costly) skills and expertise analysed all the software, hardware, and automated equipment on your site, and installed appropriate software patches and arranged for the hardware to be replaced? Where should you turn for advice ${ }^{12}$

The millennium, and the computer problems associated with it, will be with us sooner than many of us expect or wish. It is worth imagining now what it might be like being on duty covering the accident and emergency department as the revellers are brought in from their New Year's Eve parties with no laboratory service, no working lifts, bleep systems, or telephones.

J RASHBASS

Clinical Director of Biomedical Computing,

Honorary Consultant Haematopathologist,

Cambridge University,

Addenbrooke's Hospital,

Box 111, Hills Road

Cambridge CB2 2SP, UK

email:jem@cbcu.cam.ac.uk

1 NHS executive year 2000 home page. http://www.ctf.imc.exec.nhs.uk/ publishd $/ 2000 / 2000 . h t m l$

2 Tackling the year 2000 . Department of Trade and Industry and Central Computer and Telecommunications Agency, Volumes 1-6. Cambridge: Computer and Telecommunication
Cambridge Publishers Ltd, 1997.

3 Celko J, Celko J. Double zero. Byte 1997;22:89-96.

$4 \mathrm{http}: / /$ www.yahoo.com/Computers_and_Internet/Year_2000_Problem 5 Institute of Electrical Engineers advice on embedded systems. http:// www.iee.org.uk/2000risk

6 The Millennium Bug. Economist 1997;345:25-9. 